\title{
Sequence variation of SARS-CoV-2 spike protein may facilitate stronger interaction with ACE2 promoting high infectivity
}

\section{Masaud Shah}

Department of Physiology, Ajou University School of Medicine, Suwon, Republic of Korea

\section{Bilal Ahmad}

Department of Molecular Science and Technology, Ajou University, Suwon, Republic of Korea

\section{Sangdun Choi}

Department of Molecular Science and Technology, Ajou University, Suwon, Republic of Korea

Hyun Goo Woo ( $\nabla$ hg@ajou.ac.kr)

Department of Physiology, Ajou University School of Medicine, Suwon, Republic of Korea

\section{Research Article}

Keywords: COVID-19, mAbs, Mutation, Neutralization, SARS-CoV-2, Spike, South Korea

Posted Date: March 17th, 2020

DOI: https://doi.org/10.21203/rs.3.rs-16932/v1

License: (c) (i) This work is licensed under a Creative Commons Attribution 4.0 International License. Read Full License

Version of Record: A version of this preprint was published on November 12th, 2020. See the published version at https://doi.org/10.1016/j.csbj.2020.11.002. 


\title{
Sequence variation of SARS-CoV-2 spike protein may facilitate stronger interaction with ACE2 promoting high infectivity
}

\author{
Masaud Shah ${ }^{\mathrm{a}}$, Bilal Ahmad ${ }^{\mathrm{b}}$, Sangdun Choi ${ }^{\mathrm{b}}$, Hyun Goo Woo ${ }^{\mathrm{a} *}$ \\ a. Department of Physiology, Ajou University School of Medicine, Suwon, Republic of Korea \\ b. Department of Molecular Science and Technology, Ajou University, Suwon, Republic of \\ Korea
}

*Corresponding author: Hyun Goo Woo, M.D., Ph.D., Department of Physiology, Ajou University School of Medicine, 164 Worldcup-ro, Yeongtong-gu, Suwon, Korea; Tel: 82-31-2195045, Fax number: 82-31-219-5049, E-mail address: hg@ajou.ac.kr. 


\begin{abstract}
Severe acute respiratory syndrome coronavirus 2 (SARS-CoV-2), which causes coronavirus disease (COVID-19), is a novel beta coronavirus emerged in China in 2019. Coronavirus uses spike glycoprotein to interact with host angiotensin-converting enzyme 2 (ACE2) and ensure cell recognition. High infectivity of SARS-CoV-2 raises questions on spike-ACE2 binding affinity and its neutralization by anti-SARS-CoV monoclonal antibodies (mAbs). Here, we observed Val-toLys417 mutation in the receptor-binding domains (RBD) of SARS-CoV-2, which established a Lys-Asp electrostatic interaction enhancing its ACE2-binding. Pro-to-Ala475 substitution and Gly482 insertion in the AGSTPCNGV-loop of RBD hindered neutralization of SARS-CoV-2 by anti-SARS-CoV mAbs. In addition, we identified unique and structurally conserved conformational-epitopes on RBDs, which can be potential therapeutic targets. Collectively, we provide new insights into the mechanisms underlying the high infectivity of SARS-CoV-2 and development of new effective neutralizing agents.
\end{abstract}

Key Words: COVID-19, SARS-CoV-2, peptide vaccine, receptor binding domain 


\section{Introduction}

SARS-CoV-2 causes coronavirus disease (COVID-19), which initially emerged in Wuhan city in China, and has rapidly transmitted to almost every corner of the world within two and a half months $(1,2)$. Phylogenetic analysis has confirmed that SARS-CoV-2 is a novel beta coronavirus $(3,4)$ and apparently transmits from human-to-human markedly faster than previously known coronaviruses, such as SARS-CoV and MERS-CoV $(5,6)$. Although the epidemiological features of SARS-CoV-2 are largely unknown, asymptomatic transmission and poor self-quarantine measurements of infected persons are thought to be the most crucial reasons for the uncontrolled spread. In the current pandemic situation of SARS-CoV-2, there is an urgent need to develop effective therapeutics and vaccines. Several pre-existing anti-viral drugs are now under clinical trials (2).

Among the mode of action mechanisms of the viral infection, cell recognition and entry of virus are the most crucial steps that determine viral infectivity and pathogenesis (7). Coronaviruses uses spike (S) glycoprotein to interact with the human respiratory and epithelial cells expressing angiotensin-converting enzyme 2 (ACE2) receptors $(8,9)$. The ectodomain of $\mathrm{S}$ protein is an $\sim 1200$ amino acid long trimeric class 1 fusion protein and normally exists in a metastable prefusion conformation of "laying or down", which can undergo conformational rearrangement and acquire an ACE2-feasible conformation, i.e., "up or standing" $(9,10)$. The "laying or down" and "up or standing" poses are differentiated due to the conformational rearrangement of the receptor binding domain (RBD, 200 amino acid) in the $\mathrm{S} 1$ subunit of the $\mathrm{S}$ protein. The RBD contains a receptor-binding determining region (RBDR) that recognizes ACE2. The availability of RBDR is controlled by the hinge-like conformational motion of the RBD (9). Thus, S protein is indispensable for the virus survival and remains a priority target for antibodies to curb viral entry. Although a recent report has shown an arrangement of the SARS-CoV-2 S protein domains through Cryo-EM, the structure was not complete and lacked many crucial loop regions that are responsible for receptor and antibody binding (PDB ID: 6VSB) (9). In this study, we constructed a full-length model of the SARS-CoV-2 $\mathrm{S}$ protein in its pre-fusion monomeric and trimeric conformation. By using the ACE2-binding structures of SARS-CoV RBD (sRBD) and SARSCoV-2 RBD (cRBD), we investigated their relative dynamic interaction, stability, and binding affinities. In addition, we identified conformational epitopes on the cRBD that must be taken into 
account while investigating the cross-reactivity of anti-sRBD mAbs or designing new mAbs and peptide vaccines against SARS-CoV-2 S protein.

\section{Methods}

\section{SARS-CoV-2 spike protein and antibody modeling}

The full-length monomeric and trimeric $S$ protein models were generated using multiple SARSCoV structures as template, retrieved from Protein Data Bank (PDB) (PDB ID: 5X5B, 6ACG, and 5I08). The monomeric spike proteins were assembled into two conformational states based on the position of RBD (standing or laying). The amino acid sequence used in SARS-CoV-2 S modeling was retrieved from NCBI (accession \# NC_045512). Modeling procedures for protein-protein docking and interface analyses were performed as previously described (11-13). Protein surface and patch analyses were performed in Molecular Operating Environment (MOE) suit (2019.0102) as described previously (14).

For the CR3014 and CR3022 mAbs modeling, a built-in MOE suit was used and the single chain variable fragments ( $\mathrm{scFv}$ ) were constructed (15). The complementarity-determining regions (CDR) were annotated and numbered according to Chothia and Lesk numbering scheme $(16,17)$. Structural data of other mAbs including 80R, m396, F26G19 and s230 were obtained from PBD (18-21). For mAbs docking, a built-in protein-protein docking procedure was used in MOE suit. In docking simulation, CDR regions of mAbs were considered as ligand-sites instead of entire $\mathrm{scFv}$ regions.

Conformational epitopes of cRBD were predicted using Epipred implemented in SAbPred web server $(22,23)$. Briefly, this tool utilizes CDR information of an input mAb and predicts conformational epitopes on a target protein. By calculating geometric fitting and knowledge-based asymmetrical antibody-antigen scoring, the epitopes of the cRBD were predicted and ranked on the basis of combined conformational matching of the antibody-antigen structures. The score of the epitope is calculated using the following formula:

\section{Epitope Score $=\sum_{\underline{n} \underline{\underline{G}}} d(n) \operatorname{Pr}\left(T_{a b}, T_{a g}\right)$}

where $T_{a b}$ and $T_{a g}$ are the amino acid types of the antibody and antigen residues, respectively, which belong to node $n$. 


\section{Molecular dynamics (MD) simulations and binding free energy analysis}

MD simulations were performed using GROMACS 2019.3. The RBD-ACE2 complexes were solvated with TIP3P water cubic box of dimension boundaries extended to $10 \AA$ from protein atoms. To neutralize the charge of the simulation system the $\mathrm{Na}^{+} / \mathrm{Cl}^{-}$counter ions were added and energy minimization was performed using CHARMM37 force field (24) and steep descent algorithm. After temperature and pressure equilibration, MD simulations were carried out for 30 ns for each system. A detailed procedure has been described in our previous studies $(12,14)$. Free energy of RBD-ACE2 complexes was calculated using molecular mechanics of MM-PBSA approach (25). The enthalpy of the system was also calculated using MM-PBSA. The effect of polar and non-polar part of the solvent effect on free energy was determined using the PoissonBoltzmann equation and calculating the surface area. The basic equation is given as follows:

$$
\Delta G_{\text {bind }}=\Delta E_{M M}+\Delta \Delta G_{\text {sol }}-T \Delta S
$$

Where MM stands for Molecular Mechanics and sol stands for solvation. T $\Delta \mathrm{S}$ is normal mode analysis to determine the loss of flexibility upon binding.

In GROMACS, the built-in tool g_mmpbsa and APBSA were called for the MMPBSA calculations, and the last $10 \mathrm{~ns}$ of MD simulations trajectory of each complex with 1000 frames in each trajectory were extracted for energy calculations. For g_mmpbsa analysis, the dielectric constant of the aqueous solvent was set to 80 , and the interior dielectric constant was set to 4; the surface tension constant $\mathrm{g}$ was set to $0.022 \mathrm{~kJ} / \mathrm{mol}$.

\section{Results and Discussion}

\section{Structural modeling of the SARS-CoV-2 spike and ACE2 interaction}

A full-length S protein is composed of S1 and S2 subunits, which are further divided into subdomains with distinct functions. Based on the hinge-like motion of the RBD of S1 subunit, the trimeric S protein exists as transiently symmetric (RBD down) or asymmetric conformation (RBD standing) (Figure 1A). Recent studies with Cryo-EM analysis revealed consistent results that the cRBD, like other coronaviruses, exhibit stochastic breathing-like movement, facilitating receptor binding to the exposed RBD and subsequent shedding of the S1 subunit $(9,10)$. However, this trimeric structure may not be helpful in understanding the receptor binding mechanisms, because structural information on the residues in RBDR and mAbs-binding loops are missing from the 3D structure deposited at PDB (Supplementary Figure 1A, PDB ID: 6VSB). Thus, by using SARS- 
$\mathrm{CoV} \mathrm{S}$ protein structures, we constructed monomeric and trimeric models of the SARS-CoV-2 S protein with different conformational states. The structures of trimeric models are provided in the supplementary data. To understand the structure more clearly and validate our structure modeling, the Cryo-EM structure and our models were superimposed in standing (in the presence of ACE2) and laying conformation, respectively. Total root mean square deviation (RMSD) of the backbone atoms of $\mathrm{S}$ protein in standing pose was $2.93 \AA$ while that of the RBD region was $1.45 \AA$ (Supplementary Figure 1A). By contrast, RMSD values in the laying pose were $2.56 \AA$ and 1.38 $\AA$, respectively. This validation supports the reliability of our model and further suggests that the structure modeled by Cryo-EM may not be able to capture the loop structure from the stochastic motion of RBD in standing pose, which is critical for binding of receptors and antibodies.

During the preparation of our manuscript, Yan R et al. demonstrated the interaction between SARS-CoV-2 RBD and ACE2 by performing Cryo-EM analysis (26). Although, the findings of this study were consistent with our results and supported the reliability of our computational model, the structure-related information were not yet available at the PDB. Another study, which is currently under review, has delineated the interface of ACE2 with a chimeric RBD through X-ray crystallography (27). Overall this chimeric structure resembles our model and recently reported Cryo-EM structure (26) and validates the cRBD-ACE2 interface residues in our model; nonetheless, the chimeric structure lacks the crucial Val-to-Lys417 mutation. This is because, the core (scaffold) RBD in this structure was taken from SARS-CoV which is considerably conserved between the two viruses with few mutations including Val-to-Lys417 (Supplementary Figure 1B). Therefore, we suggest that our computational model provides better understanding about the structural variation in cRBD and its interface with ACE2.

By performing protein patch analysis, we demonstrated that the standing cRBD exposes Lys417 that establishes transient but strong electrostatic interaction with Asp30 of the ACE2, although this patch remains buried in the laying position of the cRBD (Figure 1B). This finding indicates that the Lys417 mutation in cRBD plays a crucial role in ACE2 recognition that is otherwise substituted by hydrophobic valine in SRBD (Figure 1C). In addition, sequence and structure analyses revealed that RBDR of SARS-CoV-2 was substantially variable compared to that of SARS-CoV, which harbored few conserved motifs. The average RMSD for the whole cRBD and sRBD was $\sim 1.1 \AA$, whereas the average RMSD for RBDR deviated by $\sim 2-3 \AA$ owing to the glycine insertion and other mutations. Both cRBD and sRBD established similar contacts with ACE2 residues (Table 1), 
although their RBDR sequences were highly variable. Initial docking analysis revealed that the electrostatic contact between Arg426 and Glu329 in sRBD-ACE2 was analogous to that of Lys417 and Asp30 contact in cRBD-ACE2 (see Figure 1D). However, this interaction was transient and break after the Asp30 of ACE2 established an intrachain contact with the nearby His34. The 3D structure of cRBD-ACE2 complex has been provided in the supplementary data.

\section{Mutation of Lys417 in cRBD may facilitate stronger interaction with ACE2}

Differing from SARS-CoV and SARS-related CoVs, the S protein of SARS-CoV-2 has furin cleavage site at the S1/S2 boundary, but exhibits similar binding affinity towards ACE2, which might be responsible for the efficient spread of SARS-CoV-2 (28). In addition to these two points, we next sought to identify sequence mutations in CRBD which play critical roles in the stronger binding-tendency towards ACE2 as compared to sRBD. As the static conformation of a protein complex provides limited information regarding the changes of the binding interface in physiological condition, we simulated the complex structure of cRBD-ACE2 and compared this with the sRBD-ACE2 complex. The distances between interface residues were monitored as a function of time to trace the shifting, breaking, or formation of new bonds. Previously, surface plasmon resonance (SPR) and bio-layer interferometry (BLI) analyses have shown that cRBDACE2 interaction is stronger than sRBD-ACE2 interaction $(9,28,29)$. Supporting this, we also observed that the total number of hydrogen bonds remained similar throughout the simulation time in both sRBD-ACE2 and cRBD-ACE2 complexes (Figure 2A). This result may imply that the stronger binding affinity of cRBD toward ACE2 might be attributed to stronger Lys417-Asp30 interaction compared to Arg426-Glu329 interaction in sRBD-ACE2. Interestingly, when we monitored the minimum interaction distances with respect to the simulation time, we observed that Lys417-Asp30 pair was more compact as compared to the Arg426-Glu329 pair. Initially the residues in both pairs were $\sim 1.4 \AA$ apart; however, the Arg426-Glu329 pair separated by $2.6 \AA$, but the Lys417-Asp30 pair remained intact till the midpoint of the simulation. The bonds between both pairs broke at the same time point and remained separated by $\sim 5 \AA$ till the end of simulation

(Figure 2A). These strong yet transient electrostatic contacts can partly explain the phenomena of receptor recognition and $\mathrm{S} 1$ shedding. $\mathrm{S}$ protein transiently utilizes the RBD of $\mathrm{S} 1$ subunit for receptor recognition and sheds them during cell internalization. Thus, faster SARS-CoV-2 transmission as compared to SARS-CoV is, at least in part, facilitated by the robust Lys417-Asp30 
interaction. In addition, we observed that Tyr449, Tyr489, Gln493, and Asn501 in cRBD established strong hydrogen bonds with the interface residues of ACE2 and remained intact throughout the simulation (Figure 2B). These results indicate that these residues are equally responsible for the relatively stronger interaction with ACE2. To demonstrate our results more clearly, we captured the motions of these interface residues in animations, and calculated binding free energies for each complex along the simulation time (Supplementary movies 1 and 2). The polar solvation energy of cRBD-ACE2 was almost half that of sRBD-ACE2, which may compensate the difference in the electrostatic energies of these complexes, resulting in overall similar total binding free energies (see Figure 2A). Collectively, our structural modeling analyses could demonstrate stronger cRBD-ACE2 interaction compared to sRBD-ACE2 interaction. In addition, we demonstrated that the mutation of Lys417 in RBDR in cRBD allowed to bind ACE2 more readily, which may facilitate the rapid transmission of SARS-CoV-2 compared to SARSCoV.

\section{Peptide vaccine can block SARS-CoV-2_ACE2 interaction}

Previously, information regarding the of sRBD-ACE2 interface has been utilized to design peptide vaccines that are able to block the receptor binding of the virus. The peptide, $\mathrm{S}_{471-503}$, derived from the ACE2 binding region of the sRBD has been able to hinder ACE2-RBD interaction and thus viral entry into the cell, as confirmed in vitro (30). Another peptide, constructed by the glycine linkage of two separate segments of ACE2 has been able to exhibit efficient antiviral activity $\left(\mathrm{IC}_{50}=0.1 \mu \mathrm{M}\right)(31)$. By comparing the cRBD region corresponding to the $\mathrm{S}_{471-503}$ (ALNCYWPLNDYGFYTTTGIGYQPYRVVVLSFEL) peptide, we found that the N-terminus (bold letters) of this peptide and corresponding cRBD region were considerably different; however, the C-terminus portion (non-bold letters) was $100 \%$ identical to cRBD (see Figure 1C). Owing to the difference in the N-terminal half, $\mathrm{S}_{471-503}$ may not hinder SARS-CoV-2 cell entry as it exhibited in SARS-CoV. Alternatively, we suggest that a peptide, HW1 (peptide sequence not disclosed yet) may abolish the interaction between cRBD and ACE2. We observed that cRBD and sRBD interact with the overall same helical peptide of the ACE2 with some differing interface residues from the RBDs (see Figure 2B and Table 1). Hence, we suggest that P6 peptide (EEQAKTFLDKFNHEAEDLFYQSS-G-LGKGDFR), reported by Han DP et al., (30) may block 
the cell entry of SARS-CoV-2 similar to that of SARS-CoV. Indeed, peptides-based vaccines are easy to design, cheaper to synthesize and faster to investigate as compared to mAbs.

\section{Identification of epitopes on cRBD that bind to SARS-CoV-2 mAbs}

SARS-CoV-2 and SARS-CoV belong to the genus betacoronavisus of the family coronaviridae and share considerable sequence identity in the $\operatorname{RBD}$ region of $\mathrm{S}$ protein $(5,28)$, allowing researchers to delineate the cross-reactivity of anti-sRBD mAbs with cRBD $(9,29)$. Although the data for RBD structure is not fully established yet, SARS-CoV-2 shares a conserved RBD with SARS-CoV, which contains drastic mutations in the RBDR regions (see Figure 1C). Thus, to estimate whether the previously known SARS-CoV mAbs can neutralize cRBD, we investigated the binding potential of the cRBD with the SARS-CoV mAbs including 80R (18), m396 (20), F26G19 (19) , s230 (21), CR3014, and CR3022 (32). The structures of mAbs were obtained from PDB [for 80R (18), m396 (20), F26G19 (19), and s230 (21)] or modeled them [for CR3014 and CR3022 (30)](for details see Methods; the 3D structures of these mAbs are provided in

Supplementary data). The variable heavy ( $\mathrm{VH}$ ) and variable light (VL) chains of scFv regions in these mAbs were aligned and their CDRs were annotated (Figure 3A). These models revealed that the VL-CDR1 of CR3022 and s230 was relatively longer and more similar as compared to the CDR1 of the other mAbs in their VL chain. In addition, the VH-CDR3 of s230 was more expanded than those of the other mAbs (Figure 3B). Differences in the sequence and length of the CDRs indicate that these mAbs recognize distinct epitopes on the RBD and may not overlap thoroughly.

In addition, we predicted three conformational epitopes of cRBD using the structural information of the mAbs. To ensure the authenticity of the epitope prediction, the co-crystal structure of sRBD-F26G19 was used as control. We observed that epitope 1 completely overlapped with the experimental result, supporting the reliability of our analysis (Figure 3C). Among the predicted cRBD epitopes, the residues in epitope 2 were mainly composed with highly variable regions between sRBD and cRBD. In contrast, the residues of the epitope 1 and 3 were significantly conserved between sRBD and cRBD (epitope 1,93\%; epitope 3 and 100\%, Figure 3D). This result implies that the anti-SARS-CoV sRBD mAbs recognizing epitope 1 or epitope 3 can neutralize the cRBD in SARS-CoV-2. However, the epitope 2 region was highly variable between cRBD and sRBD, therefore the anti-sRBD mAbs recognizing epitope 2 may not be able to recognize cRBD. 


\section{Highly conserved epitope 3 of cRBD is a promising target for anti-SARS-CoV-2 agents}

Recent studies comprising SPR and BLI analyses have demonstrated that the sRBD mAbs including m396, 80R, s230, and CR3014 cannot recognize cRBD (9, 29). However, these studies did not show whether the interface residues of these mAbs can hinder their binding to cRBD. To evaluate this, we placed or docked the $\mathrm{scFv}$ regions of these sRBD mAbs into cRBD revealing their interface residues (Table 2). s230 and 80R interacted with a part of the overlapping residues at the hypervariable RBDR region of cRBD, while m396 and F26G19 were partly overlapped with the residues at non-epitope regions (Figure 4A). These findings indicate that m396 and F26G19 cannot bind and neutralize cRBD. The binding affinity of F26G19 with cRBD has not been studied yet by SPR or BLI analyses, requiring further evaluation in near future. In addition, we found that epitope 2 on cRBD harbored a mutated loop region (475-AGSTPCNGV-483) that has been reported to abrogate the binding of CR3014 mAb to sRBD (32). Taken together, we suggest that these m396, 80R, s230, and F26G19 mAbs recognize non-conserved or non-epitope regions of cRBD, and therefore might not be able to neutralize the S protein of SARS-CoV-2.

Interestingly, cRBD escapes from the anti-sRBD mAbs even though cRBD can bind to ACE2 with high affinity. This could be partly explained by the structural differences of binding regions between them. Anti-sRBD mAbs have CDR that are very specific and recognize conformational epitopes on RBD, while ACE2 utilize a long helix that binds longitudinally to RBD.

Notably, we observed a mutation at Ala475 in cRBD, which corresponded to Pro462 in sRBD (see Figure 1C). A previous study has shown that CR3014 mAb was not effective on the mutant Pro462Leu viruses, although it could prevent lung damage and SARS-CoV shedding in ferrets (32). Moreover, we found a glycine insertion mutation in the same loop (475-AGSTPCNGV-483), lengthening the loop RMSD to 2-3 $\AA$ (see Figure 1C). This might be the reason why the previous BLI study could not demonstrate the binding of cRBD with CR3014 (29).

With this concern, to further evaluate the binding of CR3014, we performed epitope mapping and protein ligand interaction fingerprints (PLIF) analyses. CR3014 was clustered around the same AGSTPCNGV-loop, a part of epitope 2, implying that the epitope 2-targeting sRBD mAbs may not be able to neutralize cRBD (Figure 4B). Likewise, the sRBD mAbs recognizing the epitopes in variable RBDR regions may also not be able to neutralize cRBD. Thus, we suggest that mAbs 
or therapeutic peptides that bind to a conserved epitope on RBD can hinder the interaction of both SARS-CoV and SARS-CoV-2 spike with ACE2.

CR3022 has been reported to completely neutralize the CR3014 escape mutants (i.e., Pro462Leu) and synergize the neutralizing effect of CR3014 without competing with its epitopes (30). This previous study has also shown that CR3022 does not compete with cRBD and has demonstrated strong binding affinity in the BLI analysis; in contrast, the other mAbs, such as CR3014, m396, and MERS-CoV neutralizing mAb m336 were not able to bind to cRBD. These results imply that CR3022 but not the other mAbs bind to a conserved epitope of RBD in SARSCoV-2 and SARS-CoV. To evaluate this, we performed an antibody docking procedure and calculated PLIF based on 100 docked poses of the CR3022-cRBD complex. Differing from CR3014, CR3022 was clustered over Arg24 and Arg26 and interacted with Glu19, which we designated as epitope 3 in the cRBD (Figure 4B, C).

Recently, Park et al. have performed computational analyses to demonstrate whether the previously known anti-MERS-CoV and anti-SARS-CoV mAbs can bind and neutralize cRBD (under review) (33). However, this study did not consider the fact that the spike protein, particularly the RBD of SARS-CoV and MERS-CoV, have significant variations, which may preclude neutralization of cRBD by anti-SARS-CoV and anti-MERS-CoV mAbs (34). In their docking analysis, the binding of CR3022 and s230 overlapped on the same interface of cRBD. In contrast, we and Tian et al. (29) have demonstrated that there is no overlap between CR3022 and the ACE2-binding region of cRBD (see Supplementary Figure 2A). A crystal structure analysis also revealed that s230 can bind to the ACE2-binding region of sRBD (21). To validate these results, we superimposed the structures of s230-sRBD and ACE2-sRBD complexes, which revealed that ACE2 and s230 were overlapped with the same interface of sRBD (Supplementary Figure 2B). We also found that CR3022 did not compete with the ACE2 and CR3014 interfaces of cRBD, which was consistent with the results of previous studies for SARS-CoV (32) and the recent SARS-CoV-2 (29) (Supplementary Figure 2A). Notably, we found that CR3022 recognized a highly conserved region, partly overlapping with epitope 3 (Figure 4C). This result strongly indicate that CR3022 has a potential, at least in part, to neutralize cRBD. Thus, we suggest that the conserved epitopes of RBD can serve as a promising targets for engineering the broadrange or cross-reactive mAbs against SARS-CoV and SARS-CoV-2. 
In summary, we suggest that Lys417 mutation in cRBD acquires stronger electrostatic interaction with ACE2, which may facilitate faster receptor-recognition of cells. This interaction is further strengthened by electrostatic and hydrophobic contacts at the cRBD-ACE2 interface. In addition, we identified a conserved epitope on RBD, which might be the target for developing new peptide vaccines or $\mathrm{mAb}$ for neutralizing SARS-CoV-2. Undoubtedly, our findings can provide new insights on the underlying mechanisms on the high infectivity of SARS-CoV-2, which may be helpful for developing new effective neutralizing agents against SARS-CoV-2.

\section{Acknowledgements}

This research was supported by grants from the National Research Foundation of Korea (NRF) funded by the Ministry of Science and ICT (MSIT) (NRF-2017M3C9A6047620, NRF2019R1A5A2026045, NRF-2017M3A9B6061509), Republic of Korea.

\section{Author contributions}

M.S. S.C, and H.W. contributed toward conceptualization and methodology; M.S. and B.A. performed the investigation and formal analysis; M.S. and H.W. wrote the original manuscript draft; H.W and S.C. supervised the study; H.W. was responsible for funding acquisition; all the authors contributed to editing and reviewing the manuscript.

\section{Competing interests}

All authors declare that there is no competing interest.

\section{Materials \& Correspondence}

Correspondence and material requests should be addressed to Hyun Goo Woo. 


\section{Figure legends}

Figure 1. Modeling of the monomeric and trimeric SARS-CoV-2 S and ACE2 binding of the receptor binding domain $(\mathrm{RBD})$.

A) Domain architecture of the monomeric full-length $\mathrm{S}$ protein and its trimeric states are shown. The RBD are displayed in receptor accessible "standing" and non-accessible "Laying" conformation. B) The surface patch analysis suggests the exposure of Lys417 in standing conformation (Asp30 belongs to ACE2 in the patch analysis). The color codes are displayed according to the charge of the patch. C) RBD alignment of the SARS-CoV-2 (accession \# NC_045512) and SARS-CoV (PDB ID: 6ACG). Residue numbers at the start are according to the full-length $\mathrm{S}$ model and the top numbers represent RBD region only. The numbers at the start are used in RBD-ACE2 docking analysis, while the top numbers are used in RBD-mAbs docking analysis. The lower panel shows the superimposition of sRBD and cRBD. The enhanced box shows crucial mutations responsible for high receptor binding (Lys417) and antibody resistance (Ala475, Gly482). D) The superimposed RBD-ACE2 complexes in both SARS-CoV and SARSCoV-2 are shown, and the interface residues are depicted as sticks. Electrostatic contacts are highlighted in boxes.

\section{Figure 2. Binding affinity and interface analysis of the RBD-ACE2 complexes.}

A) The RMSD of the backbone atoms of the RBD-ACE2 complexes with reference to the $0 \mathrm{~ns}$ conformation are shown. Total number of hydrogen bonds between RBD-ACE2 interfaces is shown on the bottom left panel. The minimum contact distances, as a function of time, between the oppositely charged residues at the RBD-ACE2 interface are shown on the top right panel. The contact distances between the interface residues of cRBD-ACE2 complex are shown. The bottom panel shows the comparative binding free energies for the RBD-ACE2 complexes. B) The interfaces (PDB frames extracted at $20 \mathrm{~ns}$ of simulation) of ACE2 with sRBD (left) and cRBD (right) and their electrostatic surface maps, respectively, are shown.

Figure 3. Epitope mapping of the cRBD and complementarity-determining region (CDR) annotation of the mAbs.

A) Anti-sRBD mAbs (single-chain variable fragments (scFv)) and their CDRs are shown. B) The variable light $(\mathrm{VL})$ and variable heavy $(\mathrm{VH})$ chains of the $\mathrm{scFv}$ regions are aligned and the 
CDR regions are annotated according to Chothia and Lesk numbering scheme. C) The epitope prediction was validated through sRBD-F26G19 complex (PDB ID: 3BGF). The tabular interface is reported in the crystal structure while red boxes in the aligned sequences show the EpiPred predicted epitope. D) Conformational epitopes predicted with reference to six known anti-sRBD $\mathrm{mAbs}$ are highlighted and encircled. Residues participating in epitopes are indicated with arrows (the arrow colors correspond to their respective epitopes).

\section{Figure 4. Interface and protein ligand interaction fingerprints (PLIF) analysis.}

A) The binding interface of mAbs are displayed with respect to the predicted epitopes on cRBD (Green $=$ Epitope 1, Cyan $=$ Epitope 2, Red $=$ Epitope 3; this has been further explained in Figure 4). B) PLIF analysis of the CR3014 and CR3022 mAbs with cRBD. C) CR3014 cluster around epitope 2, which is highly variable between sRBD (left) and cRBD (right). CR3022 cluster near epitope 3 , which is conserved between cRBD and sRBD. 


\section{References}

1. WHO. Coronavirus disease 2019 (COVID-19) Situation Report - 466 March 2020.

2. Behzadi MA, Leyva-Grado VH. Overview of Current Therapeutics and Novel Candidates Against Influenza, Respiratory Syncytial Virus, and Middle East Respiratory Syndrome Coronavirus Infections. Front Microbiol. 2019;10:1327.

3. Wu F, Zhao S, Yu B, Chen YM, Wang W, Song ZG, et al. A new coronavirus associated with human respiratory disease in China. Nature. 2020.

4. Zhou P, Yang XL, Wang XG, Hu B, Zhang L, Zhang W, et al. A pneumonia outbreak associated with a new coronavirus of probable bat origin. Nature. 2020.

5. Zhu N, Zhang D, Wang W, Li X, Yang B, Song J, et al. A Novel Coronavirus from Patients with Pneumonia in China, 2019. N Engl J Med. 2020;382(8):727-33.

6. Paraskevis D, Kostaki EG, Magiorkinis G, Panayiotakopoulos G, Sourvinos G, Tsiodras S. Full-genome evolutionary analysis of the novel corona virus $(2019-n C o V)$ rejects the hypothesis of emergence as a result of a recent recombination event. Infect Genet Evol. 2020;79:104212.

7. Gallagher TM, Buchmeier MJ. Coronavirus spike proteins in viral entry and pathogenesis. Virology. 2001;279(2):371-4.

8. Letko M, Marzi A, Munster V. Functional assessment of cell entry and receptor usage for SARS-CoV-2 and other lineage B betacoronaviruses. Nat Microbiol. 2020.

9. Wrapp D, Wang N, Corbett KS, Goldsmith JA, Hsieh CL, Abiona O, et al. Cryo-EM structure of the 2019-nCoV spike in the prefusion conformation. Science. 2020.

10. Yuan Y, Cao D, Zhang Y, Ma J, Qi J, Wang Q, et al. Cryo-EM structures of MERS-CoV and SARS-CoV spike glycoproteins reveal the dynamic receptor binding domains. Nat Commun. 2017;8:15092.

11. Park S, Shin HJ, Shah M, Cho HY, Anwar MA, Achek A, et al. TLR4/MD2 specific peptides stalled in vivo LPS-induced immune exacerbation. Biomaterials. 2017;126:49-60.

12. Junaid M, Li CD, Shah M, Khan A, Guo H, Wei DQ. Extraction of molecular features for the drug discovery targeting protein-protein interaction of Helicobacter pylori CagA and tumor suppressor protein ASSP2. Proteins. 2019;87(10):837-49.

13. Achek A, Shah M, Seo JY, Kwon HK, Gui X, Shin HJ, et al. Linear and Rationally Designed Stapled Peptides Abrogate TLR4 Pathway and Relieve Inflammatory Symptoms in Rheumatoid Arthritis Rat Model. J Med Chem. 2019;62(14):6495-511.

14. Shah M, Anwar MA, Park S, Jafri SS, Choi S. In silico mechanistic analysis of IRF3 inactivation and high-risk HPV E6 species-dependent drug response. Sci Rep. 2015;5:13446.

15. Narayanan A, Sellers BD, Jacobson MP. Energy-based analysis and prediction of the orientation between light- and heavy-chain antibody variable domains. J Mol Biol. 2009;388(5):941-53.

16. Chothia C, Lesk AM. Canonical structures for the hypervariable regions of immunoglobulins. J Mol Biol. 1987;196(4):901-17.

17. Murzin AG, Brenner SE, Hubbard T, Chothia C. SCOP: a structural classification of proteins database for the investigation of sequences and structures. $\mathrm{J}$ Mol Biol. 1995;247(4):536-40. 
18. Hwang WC, Lin Y, Santelli E, Sui J, Jaroszewski L, Stec B, et al. Structural basis of neutralization by a human anti-severe acute respiratory syndrome spike protein antibody, 80R. J Biol Chem. 2006;281(45):34610-6.

19. Pak JE, Sharon C, Satkunarajah M, Auperin TC, Cameron CM, Kelvin DJ, et al. Structural insights into immune recognition of the severe acute respiratory syndrome coronavirus $\mathrm{S}$ protein receptor binding domain. J Mol Biol. 2009;388(4):815-23.

20. Prabakaran P, Gan J, Feng Y, Zhu Z, Choudhry V, Xiao X, et al. Structure of severe acute respiratory syndrome coronavirus receptor-binding domain complexed with neutralizing antibody. J Biol Chem. 2006;281(23):15829-36.

21. Walls AC, Xiong X, Park YJ, Tortorici MA, Snijder J, Quispe J, et al. Unexpected Receptor Functional Mimicry Elucidates Activation of Coronavirus Fusion. Cell. 2019;176(5):102639 e15.

22. Dunbar J, Krawczyk K, Leem J, Marks C, Nowak J, Regep C, et al. SAbPred: a structurebased antibody prediction server. Nucleic Acids Res. 2016;44(W1):W474-8.

23. Krawczyk K, Liu X, Baker T, Shi J, Deane CM. Improving B-cell epitope prediction and its application to global antibody-antigen docking. Bioinformatics. 2014;30(16):2288-94.

24. Huang J, Rauscher S, Nawrocki G, Ran T, Feig M, de Groot BL, et al. CHARMM36m: an improved force field for folded and intrinsically disordered proteins. Nat Methods. 2017;14(1):71-3.

25. Kumari R, Kumar R, Open Source Drug Discovery C, Lynn A. g_mmpbsa--a GROMACS tool for high-throughput MM-PBSA calculations. J Chem Inf Model. 2014;54(7):1951-62.

26. Yan R, Zhang Y, Li Y, Xia L, Guo Y, Zhou Q. Structural basis for the recognition of the SARS-CoV-2 by full-length human ACE2. Science. 2020.

27. Jian Shang GY, Ke Shi, Yushun Wan, Chuming Luo, Hideki Aihara, Qibin Geng, Ashley Auerbach, Fang Li. Structural basis for receptor recognition by the novel coronavirus from Wuhan. Research Square. 2020.

28. Alexandra C. Walls1 Young-Jun Park M. Alejandra Tortorici AW ATMaDV. Structure, function and antigenicity of the SARS-CoV-2 spike glycoprotein Cell press. 2020.

29. Tian X, Li C, Huang A, Xia S, Lu S, Shi Z, et al. Potent binding of 2019 novel coronavirus spike protein by a SARS coronavirus-specific human monoclonal antibody. Emerg Microbes Infect. 2020;9(1):382-5.

30. Hu H, Li L, Kao RY, Kou B, Wang Z, Zhang L, et al. Screening and identification of linear B-cell epitopes and entry-blocking peptide of severe acute respiratory syndrome (SARS)associated coronavirus using synthetic overlapping peptide library. J Comb Chem. 2005;7(5):648-56.

31. Han DP, Penn-Nicholson A, Cho MW. Identification of critical determinants on ACE2 for SARS-CoV entry and development of a potent entry inhibitor. Virology. 2006;350(1):1525.

32. Ter Meulen J, van den Brink EN, Poon LL, Marissen WE, Leung CS, Cox F, et al. Human monoclonal antibody combination against SARS coronavirus: synergy and coverage of escape mutants. PLoS Med. 2006;3(7):e237.

33. Tamina Park. Sang LY. Seil Kim MJ, Hong GK, Sangmi Jun, Seung Kim, Bum TK, Edmond CP, Daeui Par. Spike protein binding prediction with neutralizing antibodies of SARS-CoV-2. bioRxiv. 2020. 
34. Chen Y, Rajashankar KR, Yang Y, Agnihothram SS, Liu C, Lin YL, et al. Crystal structure of the receptor-binding domain from newly emerged Middle East respiratory syndrome coronavirus. J Virol. 2013;87(19):10777-83. 
A
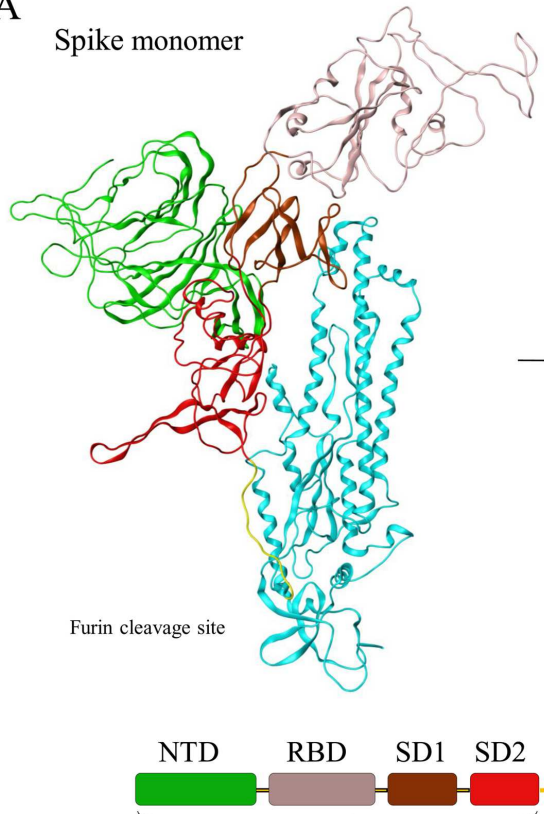

S1 Subunit

$\mathrm{C}$

336 CPFGEVFATRFASVYAWNRKRISNCVADYSVLYNSASFSTFKCYGVSPTKLNDL
323 CPFGEVFNATKFPSVYAWERKKISNCVADYSVLYNSTFFSTFKCYGVSATKLNDL 56 CF TNVYADSFVIRGDEVRQI APGQT GKI A DYNYKLPDDFTGCVIAWNSNNLDSKV CFSNVYADSFVVKGDDVRQIAPGQTGVIADYNYKLPDDFMGCVLAWNTRNIDATS $111130 \quad 150$ TGNYNYKYRYLRHGKLRPFERDISNVPFSPDGKPCT - PPALNCYWPLNDYGFYTT $\left.\begin{array}{lccl}166 & 185 & 190 \\ \text { NGVGYQPYRVVVLSFELLHAPATV } 525 & \text { SARS-CoV-2 } \\ \text { TGIGYQPYRVVVLSFELLNAPATVC511 } & \text { SARS-CoV }\end{array}\right\}$ RBD
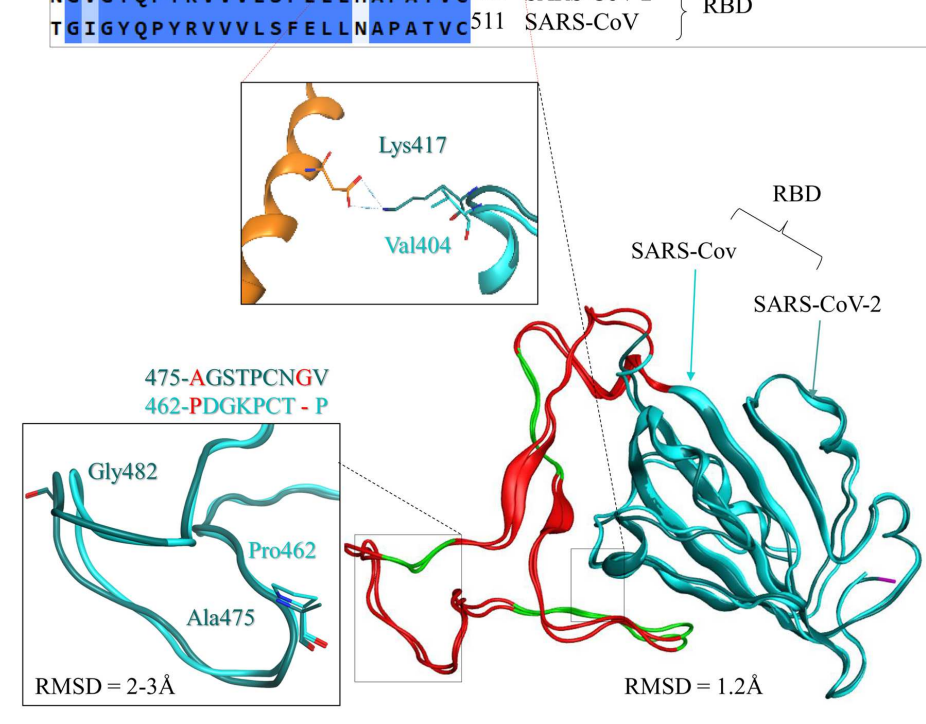

B

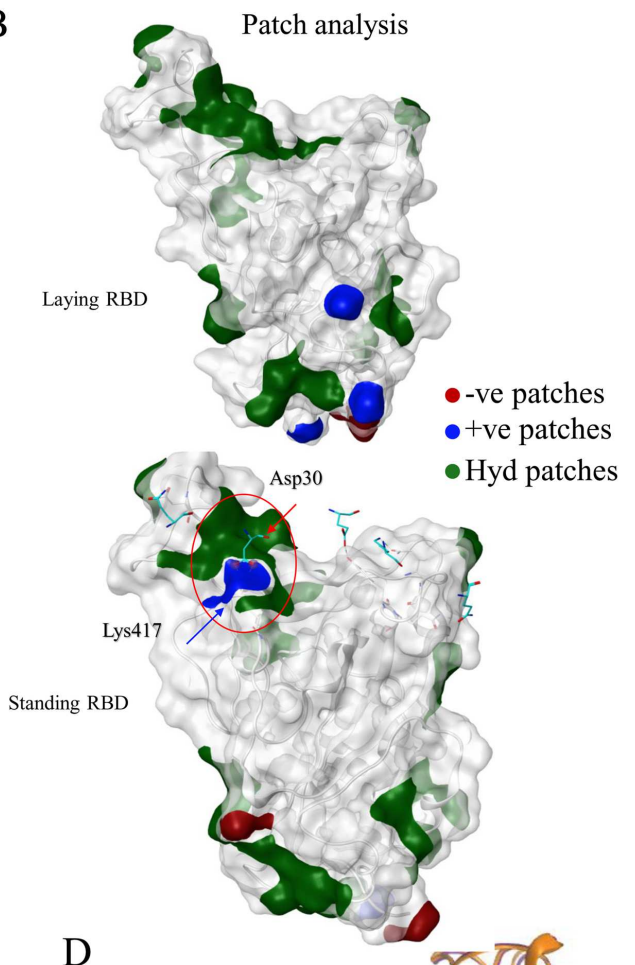

$\mathrm{D}$

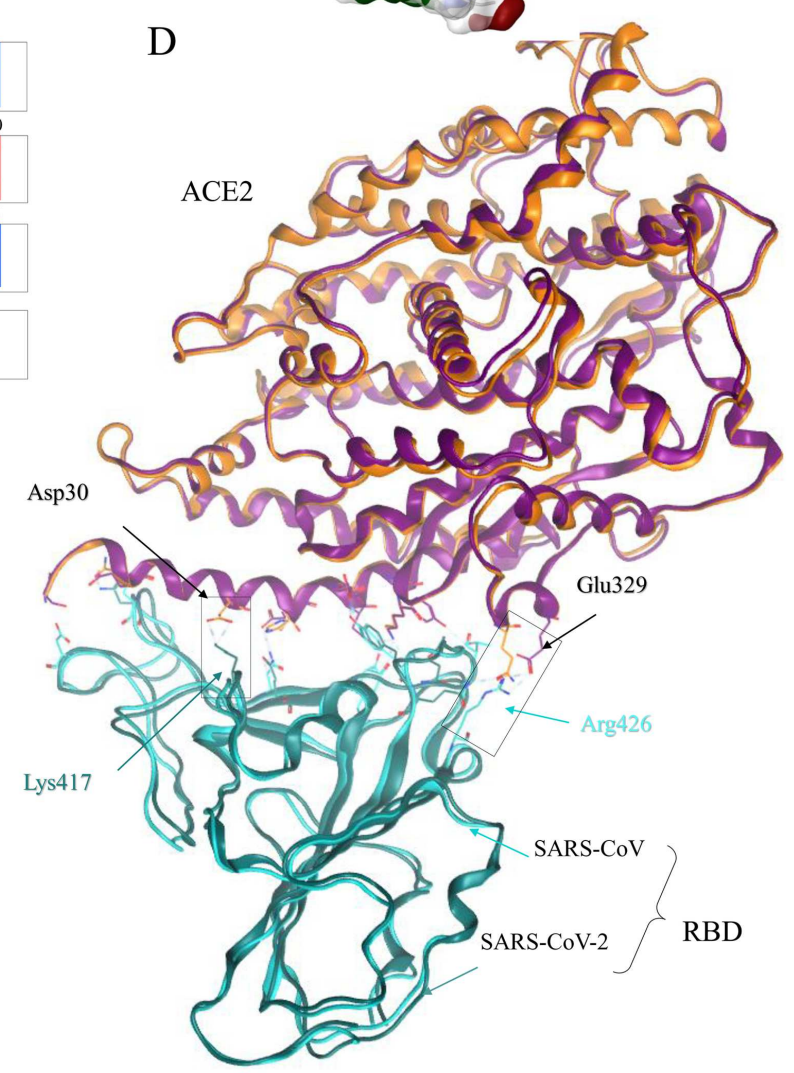

\section{Figure 1}

Modelling of the monomeric and trimeric SARS-CoV-2 S and receptor binding of the RBD. A) Domains architecture of the monomeric full-length $S$ protein and its trimeric states are shown. The RBD are displayed in receptor accessible "standing" and non-accessible "Laying" conformation. B) The surface 
patch analysis suggests the exposure of Lys417 in standing conformation (Asp30 belongs to ACE2 in the patch analysis). The color cods are displayed according to the charge of the patch. C) RBD alignment of the SARS-CoV-2 (accession \# NC_045512) and SARS-CoV (PDB ID: 6ACG). Residues number at the start are according to the full-length $S$ model and the top numbering are for the RBD region only. The numbering on the start are used in RBD-ACE2 docking analysis while the top numbering are used in RBDmAbs docking analysis. The lower panel shows the superimposition of SRBD and cRBD. Enhanced box shows crucial mutations responsible for high receptor binding (Lys417) and antibody resistance (Ala475, Gly482). D) The superimposed RBD-ACE2 complexes in both SARS-CoV and SARS-CoV-2 are shown and the interface residues are depicted as sticks. Electrostatic contacts are highlighted in boxes. 

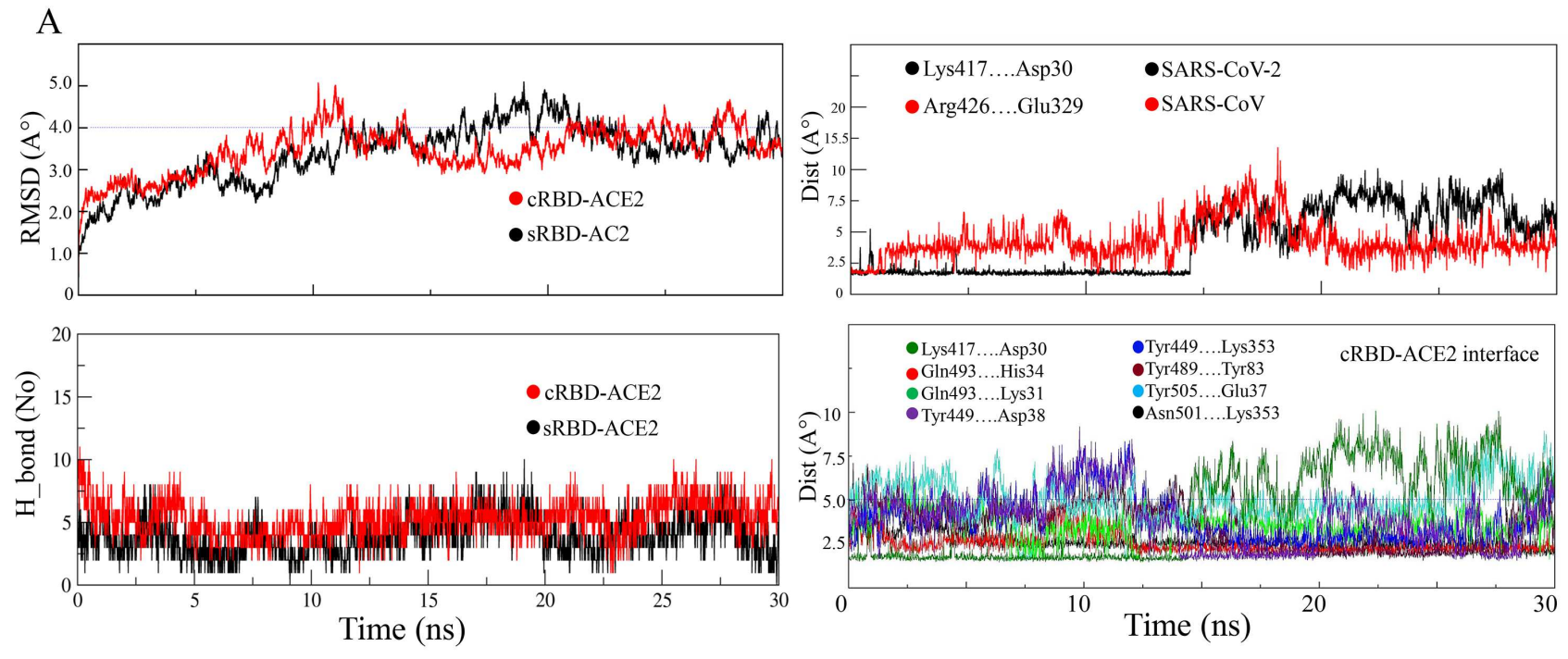

\begin{tabular}{cccccc}
\hline RBD-ACE2 & Vwd_E & Eletrostatic_E & Polar_solv_E & SASA_E & Total (kJ/mol) \\
\hline cRBD & -300.515 & -2443.874 & 344.692 & -37.66 & -2583.61 \\
sRBD & -331.853 & -2827.12 & 662.839 & -44.101 & -2639.407 \\
\hline
\end{tabular}

B
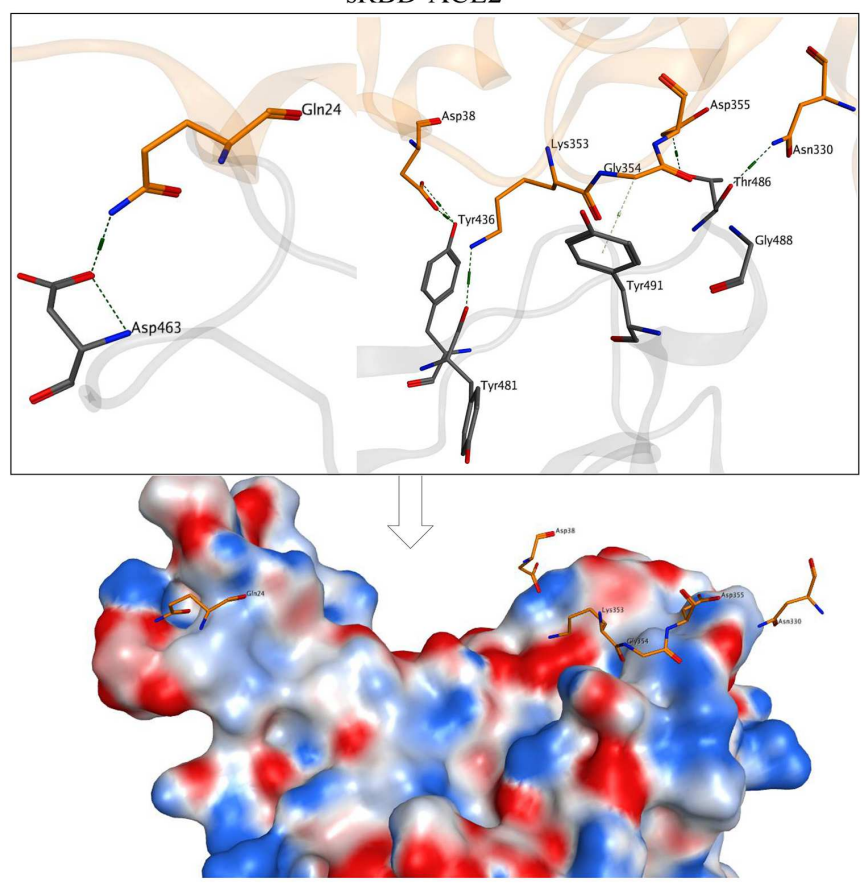

CRBD-ACE2

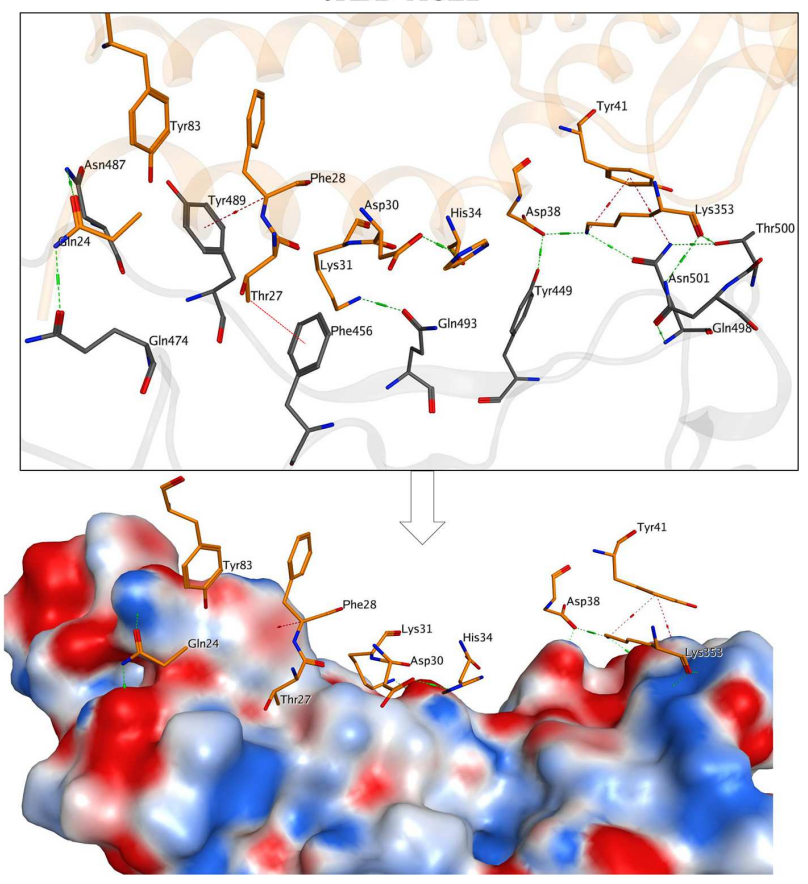

\section{Figure 2}

Binding affinity and interface analysis of the RBD-ACE2. A) The RMSD of the backbone atoms of the RBD-ACE2 complexes with reference to the 0 ns conformation are shown. Total number of hydrogen bonds between RBD-ACE2 interfaces are shown in the bottom left panel. The minimum contact distances, as a function of time, between the oppositely charged residues at the RBD-ACE2 interface are shown in the right top panel. The contact distances between the interface residues of cRBD-ACE2 complex are 
shown. The bottom panel show the comparative binding free energies for the RBD-ACE2 complexes. B) The interfaces (PDB frames extracted at $20 \mathrm{~ns}$ of simulation) of ACE2 with sRBD (left) and cRBD (right) and their electrostatic surface maps, respectively.

A

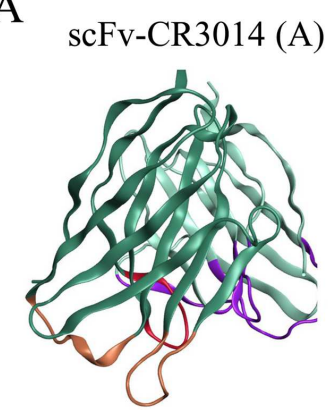

scFv-m396 (D)
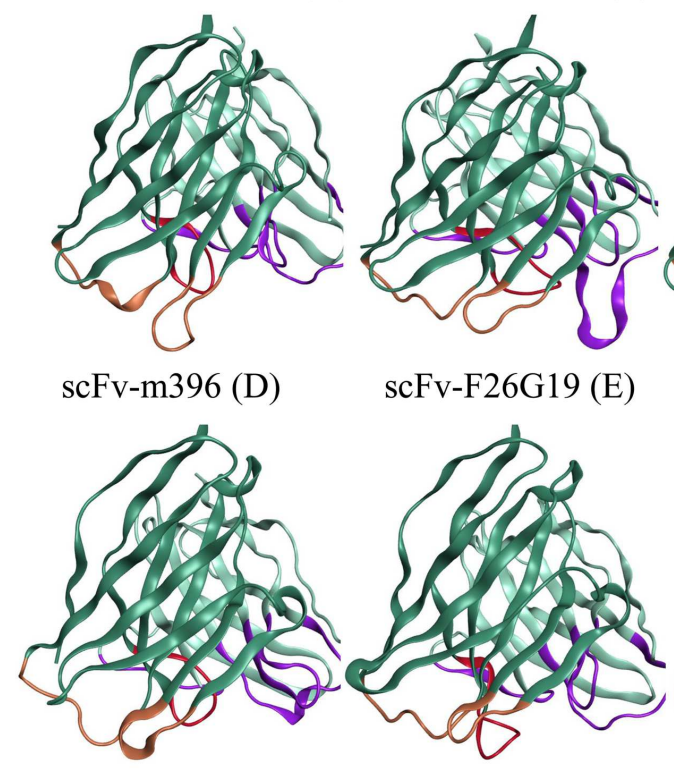

scFv-F26G19 (E)
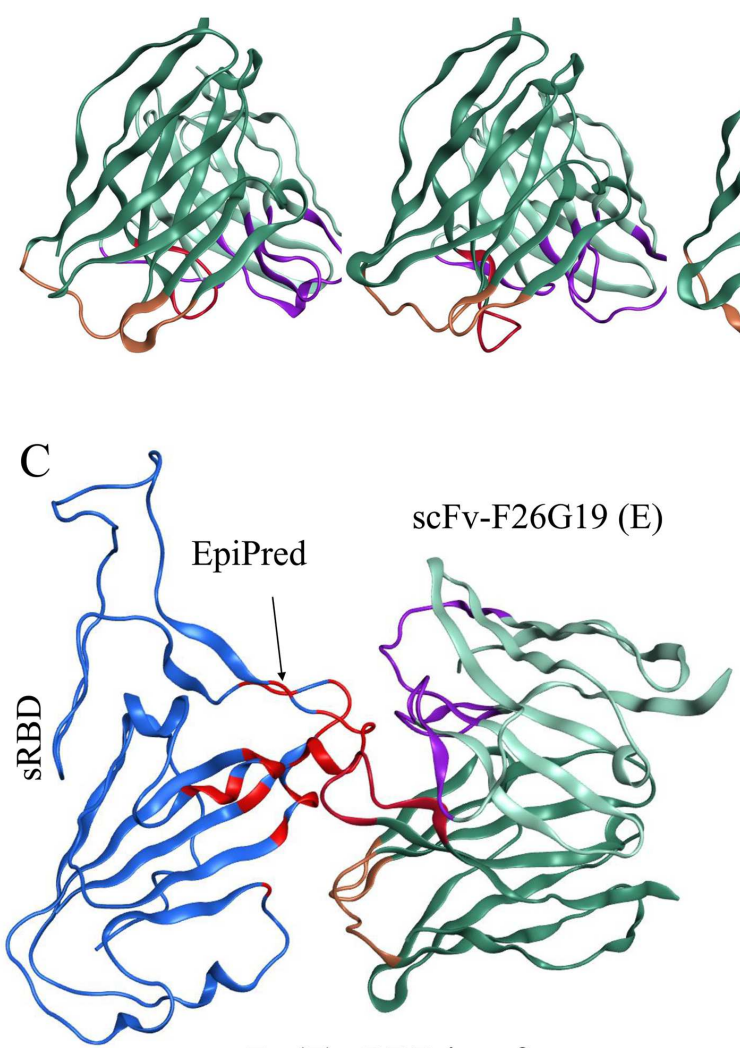

$\operatorname{scFv}(\mathrm{E})-\mathrm{sRBD}$ interface

\begin{tabular}{l|llllll}
\multicolumn{1}{l}{ sRBD } & \multicolumn{2}{c}{ E(scFv) } & Bond & E $^{\text {kcal/mol }}$ & \multicolumn{1}{c}{ Dist $\AA$} & Atom \\
\hline Ser362 & Tyr52 & H & -0.50 & 3.65 & -- \\
Asp392 & Pro98 & H & -1.10 & 3.27 & - b \\
Asp392 & Gln99 & H & -4.70 & 3.23 & - b \\
Arg426 & Asp56 & IH & -14.12 & 3.35 & -- \\
Arg426 & Thr57 & H & -1.30 & 3.42 & - b \\
Asn427 & Asp56 & H & -0.50 & 3.56 & -- \\
Thr486 & Tyr94 & H & -3.50 & 2.65 & bb \\
Gly488 & Val92 & H & -2.70 & 2.97 & bb \\
Gly490 & Gln99 & H & -0.80 & 2.66 & b-
\end{tabular}

$\mathrm{B}$

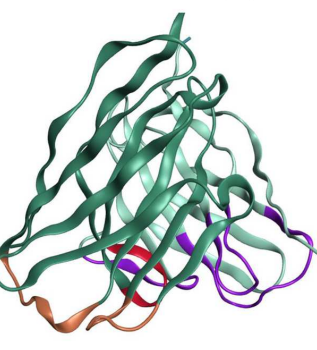

scFv-s230 (F)

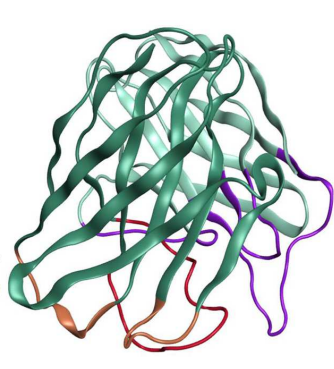

scFv VL CDR alignment

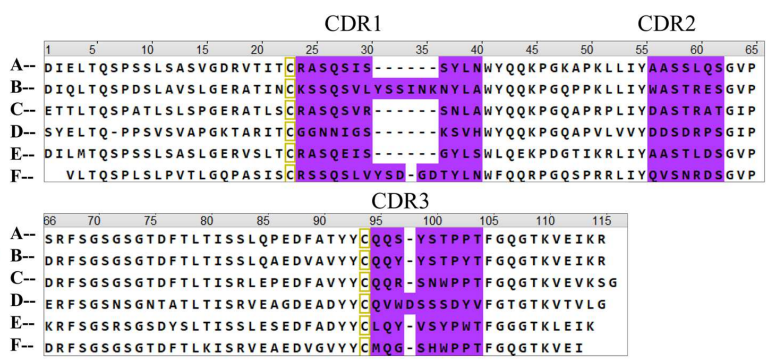

ScFv VH CDR alignment

CDR1

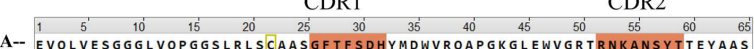
B-- EMQLVQSGTEVKKPGESLKISCKGSGYGFITYWIGWVRQMPGKGLEWMGIIYPG--DSETRYSPS C-- EVQLVQSGGGVVQPGKSLRLSCAASGFAFSSYAMHWVRQAPGKGLEWVAVISYOG.-SNKYYADS

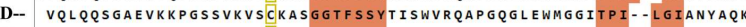
E-- EVQLEESGTVLARPGASVKMSCKASGYTFTTYRMHWIKQRPGQGLEWIGAIYPG--NSDTTYNOK

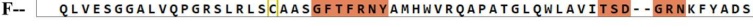

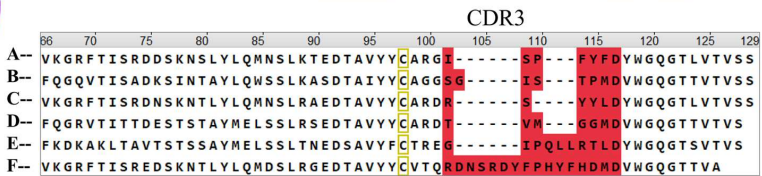

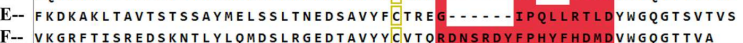

$\mathrm{D}$ Predicted Epitopes cRBD

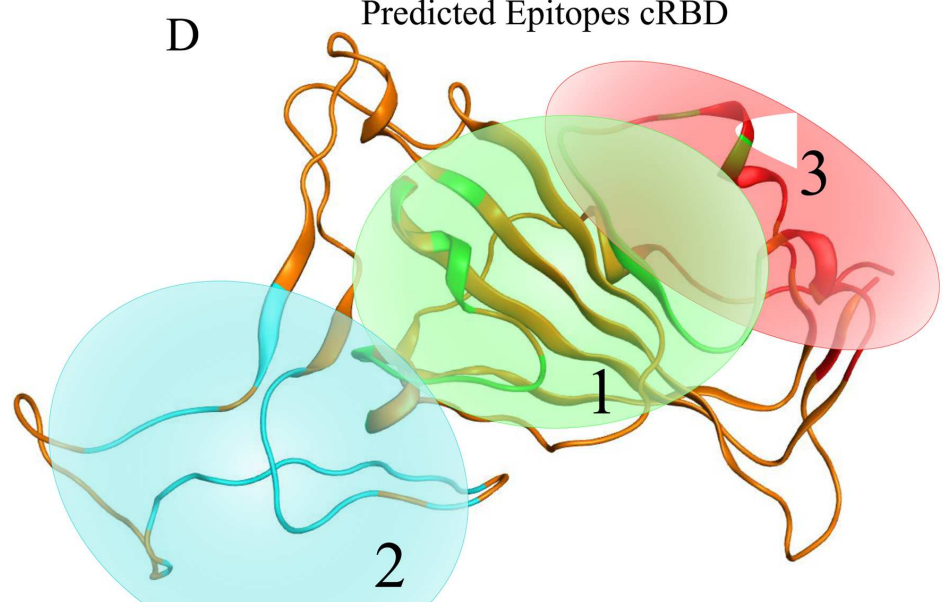

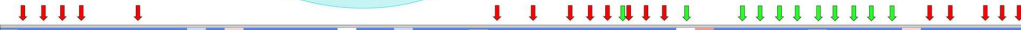
CPFGEVFNATRFASVYAWNRKRISNCVADYSVLYNSASFSTFKCYGVSPTKLNDL CPFGEVFNATKFPSVYAWERKKISNCVADYSVLYNSTFFSTFKCYGVSATKLNDL CFTNVYADSFVIRGDEVRQIAPGQTGKIADYNYKLPDDFTGCVIAWNSNNLDSKV CFSNVYADSFVVKGDDVRQIAPGQTGVIADYNYKLPDDFMGCVLAWNTRNIDATS GGNYNYLYRLFRKSNLKPFERDISTEIYQAGSTPCNGVEGFNCYFPLQSYGFQPT TGNYNYKYRYLRHGKLRPFERDISNVPFSPDGKPCT - PPALNCYWPLNDYGFYTT \begin{tabular}{|lll}
\hline N G V G Y Q P Y R V V V L S F E L L H A P A T V C & SARS-CoV-2 & $\begin{array}{l}\text { Control (sRBD) epitope } \\
\text { RBD }\end{array}$ \\
\hline Epitope 1 (cRD)
\end{tabular} \begin{tabular}{lll}
\hline TGIGYQPYRVVVLSFELLNAPATV C & SARS-CoV & RBD \\
\hline
\end{tabular}

\section{Figure 3}

Epitope mapping of the cRBD and CDRs annotation of the mAbs. A) Anti-sRBD mAbs (single-chain variable fragment scFv are shown) and their complementarity-determining regions (CDRs) are shown. B) 
The variable light $(\mathrm{VL})$ and variable heavy $(\mathrm{VH})$ chains of the scFv regions are aligned and the CDR regions are annotated according to Chothia and Lesk numbering scheme. C) The epitope prediction was validated through sRBD-F26G19 complex (PDB ID: 3BGF). The tabular interface is reported in the crystal structure while red boxes in the aligned sequences show the EpiPred predicted epitope. D) onformational epitopes predicted with reference to six known anti-sRBD mAbs are highlighted and encircled. Residues participating in epitopes are indicated with arrows (the arrow colors correspond to their respective epitopes).
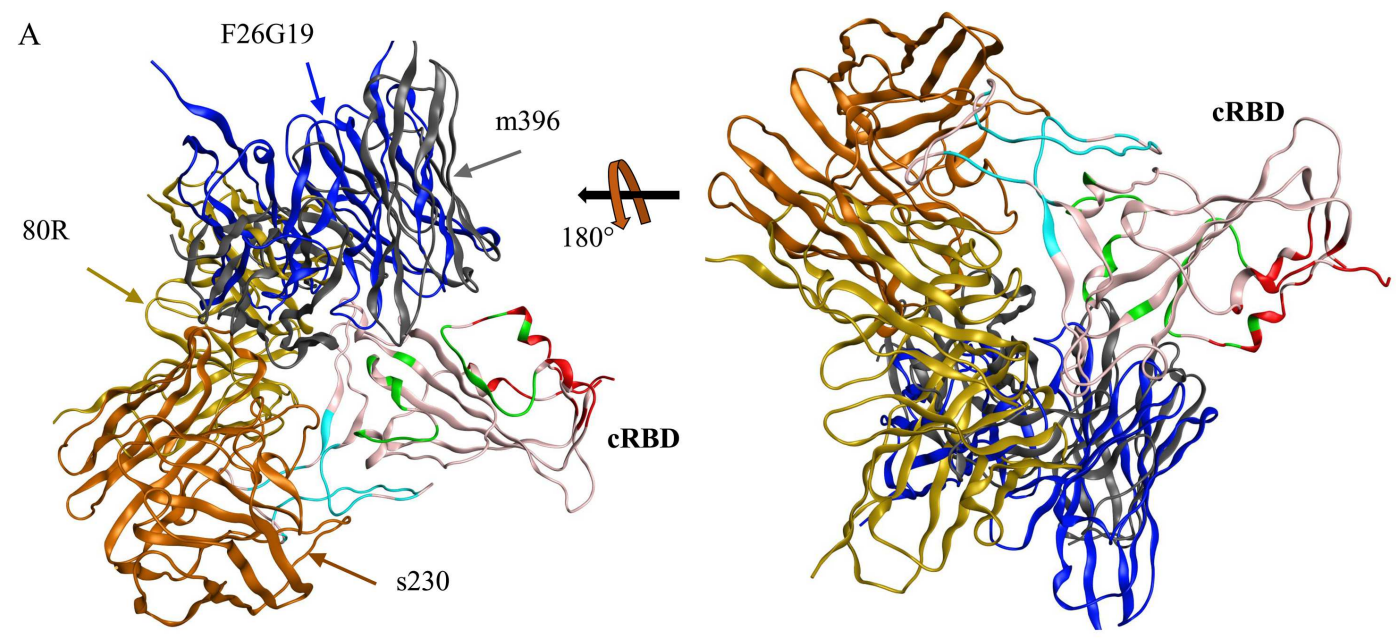

B CRBD-CR3014 PLIF
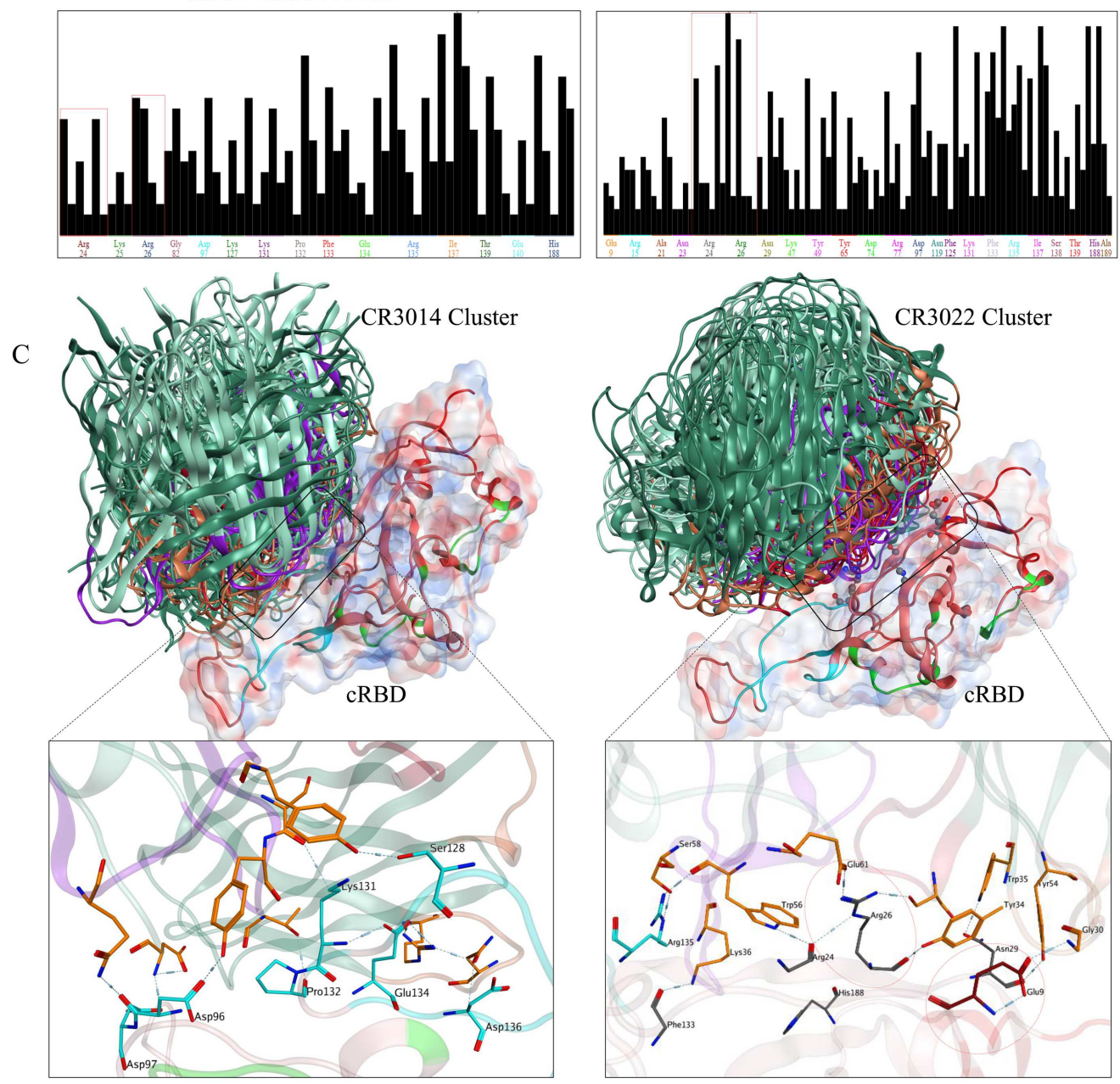


\section{Figure 4}

The interface and protein ligand interaction fingerprints (PLIF) analysis. A) The binding interface of mAbs are displayed with respect to the predicted epitopes on CRBD (Green = Epitope 1, Cyan $=$ Epitope 2, Red $=$ Epitope 3; this has been further explained in Figure 4). B) PLIF analysis of the CR3014 and CR3022 mAbs with CRBD. C) CR3014 cluster around the epitope 2, which is highly variable between SRBD (left) and CRBD (right). CR3022 cluster near epitope 3 , which is conserved between cRBD and SRBD.

\section{Supplementary Files}

This is a list of supplementary files associated with this preprint. Click to download.

- SpiketrimerACE2complex.pdb

- Spiketrimer.pdb

- CR3014mAb.pdb

- CR3022mAb.pdb

- Supplementarymovie1.m4v

- SARSCoV2SpikeACE2TABLEs.pdf

- SARSCoV2SpikeACE2Supplementary.pdf

- Supplementarymovie2.m4v

- SARSCoV2RBDACE2Complex.pdb 